\title{
A new design for imaging of fast energetic electrons
}

\author{
S. Skruszewicz ${ }^{\mathrm{a}}$, J. Passig ${ }^{\mathrm{b}}$, A. Przystawik ${ }^{\mathrm{c}}$, N.X. Truong ${ }^{\mathrm{d}}$, M. Köther $^{\mathrm{a}}$, J. Tiggesbäumker ${ }^{\mathrm{a}, *}$, \\ K.-H. Meiwes-Broer ${ }^{a}$ \\ a Institut für Physik, Universität Rostock, Universitätsplatz 3, 18051 Rostock, Germany \\ ${ }^{\mathrm{b}}$ Institut für Chemie, Universität Rostock, Dr.-Lorenz-Weg 1, 18059 Rostock, Germany

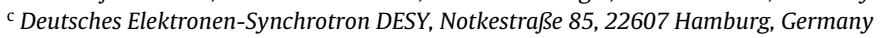 \\ ${ }^{\mathrm{d}}$ Institut für Optik und Atomare Physik, Technische Universität Berlin, Hardenbergstraße 36, 10623 Berlin, Germany
}

\section{A R T I C L E I N F O}

\section{Article history:}

Received 20 December 2013

Received in revised form 12 February 2014

Accepted 17 February 2014

Available online 25 February 2014

\section{Keywords:}

Angular distribution

Photoemission

Photoelectron spectra

Strong-field excitation

Synchrotron radiation

\begin{abstract}
A B S T R A C T
We report on an essentially improved version of the classical Eppink-Parker velocity map imaging spectrometer design (Rev. Sci. Instrum. 68, 3477 (1997)). By adding electrostatic lenses with an opposite polarity to the extraction system we succeeded in extending the range of detection of energetic particles up to the keV regime at moderate $(<20 \mathrm{kV})$ extraction voltage conditions. Simulations show that the electrostatic lens system acts in analogy to an achromatic lens in optics and leads to a reduction in the chromatic energy aberration. For comparison to other setups a transmission parameter of the extraction system is defined denoting the maximum kinetic energies of particles which can be analyzed. Detector size and spectrometer length only enter via geometry, that is the straight trajectories in the subsequent field-free particle drift. With respect to Eppink-Parker the energy range has been extended by a factor of 2.5. Moreover, particle trajectory simulations demonstrate that the energy resolution can be improved by about $20 \%$. To test the performance, photoemission studies have been conducted to resolve abovethreshold-ionization patterns from Xe atoms exposed to intense ultrashort laser pulses as well as single photon ionization of $\mathrm{Ne}$ atoms using tunable synchrotron radiation with photon energies up to $600 \mathrm{eV}$.
\end{abstract}

(C) 2014 Elsevier B.V. All rights reserved.

\section{Introduction}

The technique of Velocity Map Imaging (VMI) as introduced by Chandler and Houston [6] is a powerful and direct method to obtain a two-dimensional projection of the full particle momentum distribution. The kinetic energy and emission direction of the reaction products resulting from ionization, fragmentation, etc. are accessible in a single measurement. The widely spread Eppink-Parker setup Eppink and Parker [9] consists of a two-stage focusing electrostatic extraction and a position sensitive detector at some distance. The photoelectron trajectories leading to the momentum distribution on the detector can easily be calculated using ion trajectory simulations, e.g., SIMION8 [27]. Since its first use by Bakker et al. [1,2], the VMI-technique has found a wide range of applications in atomic and molecular physics [23,28,3] ranging up to the strong field regime [17,30], and aerosol physics [32] to only name a few. With photoelectrons, VMI spectrometers are applied to study low kinetic energies, i.e. in the range up to $60 \mathrm{eV}[14,33]$. Several modifications of the Eppink-Parker setup

\footnotetext{
* Corresponding author. Tel.: +49 3814986805.
}

have been described in the literature $[29,11,21,12]$. However, only slight improvements were achieved with respect to an extension of the mapped energies and an improved momentum resolution. For example, Garcia et al. [11] introduced slits in the repeller in order to fit the instrument to their experimental requirements at a synchrotron beamline. There are two main drawbacks, which currently limit the utilization of the classical type of spectrometer to map the angular distribution of more energetic particles:

1 For guiding and focusing of energetic particles towards the position-sensitive detector the extraction voltage has to be substantially higher compared to the particle kinetic energy. Hence one has to deal with repeller voltages largely above $10.0 \mathrm{kV}$ when trying to map keV ions/electrons. As a consequence at high gas load conditions special efforts have to be made to avoid discharges in the lens system.

2 Focusing on the detector can only be achieved within a narrow momentum range. For higher particle velocities, simulations show that the focus position substantially moves towards the extraction system. This results in a blurring of the signal on the position sensitive detector, hence a significant decrease in energy and angular resolution. 


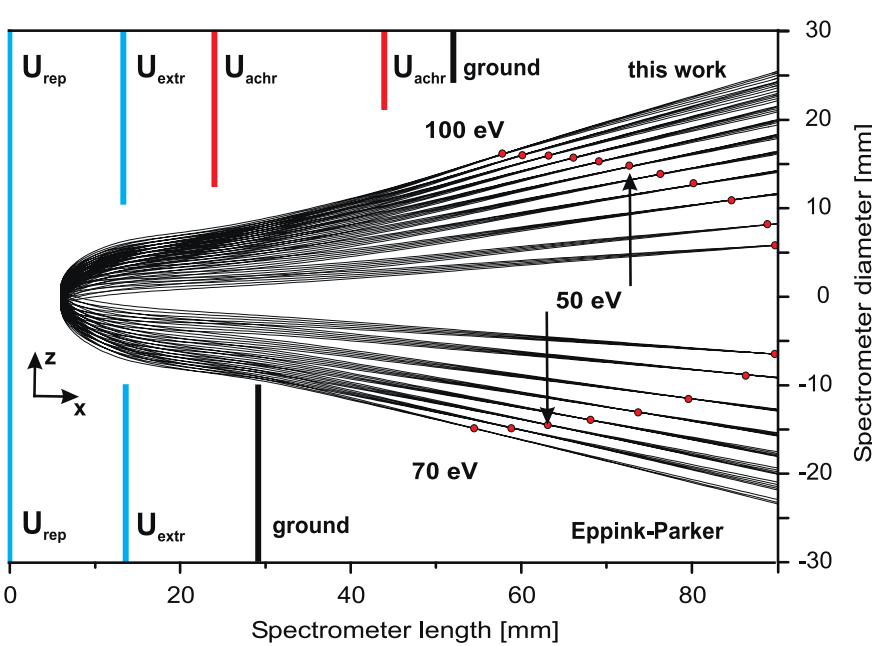

Fig. 1. Electron trajectories $\left(E_{\text {kin }}=5,10-100 \mathrm{eV}\right)$ released along a line $(x=6 \mathrm{~mm}$, $z=0 \pm 1 \mathrm{~mm}$ ) parallel to the electrodes calculated for HEVMI (top) and Eppink-Parker (bottom). A repeller voltage of $U_{\text {rep }}=-2 \mathrm{kV}$ was used in the simulations. Clearly higher energy electrons are transmitted using the HEVMI setup. Red dots indicate the calculated focal point for different energies (arrows: $E_{\text {kin }}=50 \mathrm{eV}$ ). (For interpretation of the references to color in this figure legend, the reader is referred to the web version of this article.)

To the best of our knowledge, no effort has been dedicated to extend the operational range towards keV kinetic energies. Robust VMI technique can be, however, successfully utilized in experiments at X-ray free-electron lasers [19,20], where detection of high energy electrons including angular distribution might be desirable or in intense optical laser-matter interaction studies where the fast electrons provide informations about scattering at plasmons Passig et al. [22] or the ionic center [4].

In this contribution we present a High Energy Velocity Map Imaging (HEVMI) spectrometer, which is capable to map energetic particles. Moreover the design is characterized by an improved momentum resolution when compared to the classical setup. In the following we focus in our analysis on the imaging of energetic electrons.

\section{Spectrometer design}

Generally, the operational principle of the VMI spectrometer can be regarded as a projection of the expanding Newton spheres onto a 2D position sensitive detector, whereas the geometrical size is mainly determined by the electron time-of-flight through the spectrometer. Thus an extension of the energy range can be achieved by bringing electrons as fast as possible to the detector plane. Including the impact of the focusing field of the electrostatic lenses, the system has to be optimized to enable high energy electrons to pass through the spectrometer and ensure proper focusing.

To compare the transmission of the different setups, we conducted ion trajectory calculations and relate the maximum electron energies to the repeller voltage applied, i.e. $R_{\text {rel }}$. Eppink-Parker (EP) consists of a three electrodes setup [9] and allows electrons with maximum energy $R_{\mathrm{rel}}^{\mathrm{EP}}=40 \mathrm{eV} / \mathrm{kV}$ to pass through the spectrometer without colliding with the electrostatic lens system or being significantly disturbed by field distortions near electrode edges. With the new design electrons with energies up to $R_{\text {rel }}^{\mathrm{HEVMI}}=100 \mathrm{eV} / \mathrm{kV}$ can still reach the detector. The $3 \mathrm{D}$ ion trajectory simulation package SIMION8 is used to design a compact HEVMI system. The total length of the spectrometer is reduced to $90 \mathrm{~mm}$ (from the repeller electrode to the detector plane) as shown in Fig. 1. Furthermore two positive equipotential electrodes are introduced. The extra electrodes form a concave electrostatic lens, which acts as an

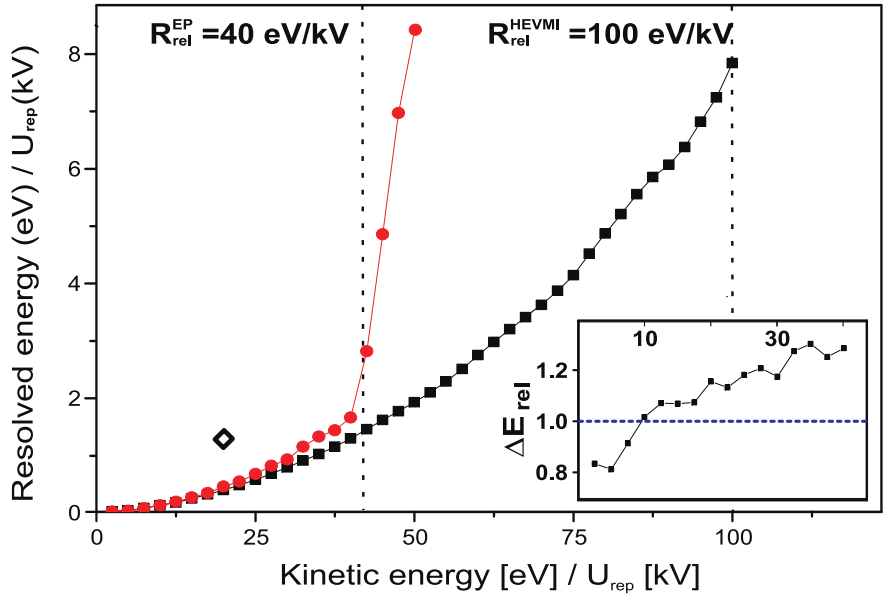

Fig. 2. Calculated energy resolution $\Delta E_{\mathrm{rel}}=\Delta E / E=2 \Delta p / p$ ( $p$ denotes the electron momentum) of Eppink-Parker (EP) $(\bigcirc)$ compared to HEVMI $(\square)$ normalized to the repeller voltage $U_{\text {rep }}$. The transmission parameter $R_{\text {rel }}$ is labeled for both setups. Above $40 \mathrm{eV} / \mathrm{kV}$ there is a distinct decrease in transmission as well as energy resolution using the EP setup. The simulations demonstrate that even near the HEVMI energy transmission limit of $100 \mathrm{eV} / \mathrm{kV}$ a value of $\Delta E_{\mathrm{rel}} \approx 0.08$ can be achieved. Experimental resolution extracted from 2 p line data shown in Fig. 5 but for an extended ionization region in $z$-direction resulting from the unfocused synchrotron X-ray beam. Inset: With respect to Eppink-Parker the simulations predict an improvement in $\Delta E_{\text {rel }}$ by about $20 \%$ for electrons faster than $10 \mathrm{eV} / \mathrm{kV}$.

achromatic doublet. In optics such an element is known to compensate for chromatic aberrations. Here uncertainties in the focus length due to different electron kinetic energies are reduced. Electron trajectories are sketched to visualize the intrinsic aberrations of the electrostatic lens system, see Fig. 1. With the achromatic lens (top) the chromatic aberration is significantly reduced compared to Eppink-Parker (bottom) resulting in an improvement in the energy resolution $\Delta E_{\text {rel }}$ of e.g. $20 \%$ above $15 \mathrm{eV} / \mathrm{kV}$, see inset in Fig. 2. For convenience $\Delta E_{\text {rel }}$ is normalized to the potential applied at the repeller plate. At higher energies $\Delta E_{\text {rel }}$ increases (Fig. 2) giving a value of 0.16 at the transmission limit of $100 \mathrm{eV} / \mathrm{kV}$. We note, that two positive electrodes instead of a single one improves the resolution by more than $15 \%$, e.g., from $\Delta E_{\mathrm{rel}}=4.2 \%$ to $4.9 \%$ at $500 \mathrm{eV}$. The calculations show that the resolution scales with $(\Delta z)^{2}$, see Fig. 1 . Hence to attain the limit experimentally, the ionization region must be restricted to one micron in $x$ and $z$-directions.

One can give a rule of thumb for the design of the HEVMI electrostatic lens system. First, the expansion of the Newton spheres for a system with mesh electrodes is simulated. This enables to neglect the rather complex influence of the focusing field on the electron trajectories. By tracing the electron pathways, this procedure gives fairly good values for the inner diameter of the electrode apertures. Next, one has to perform studies to optimize the focusing properties of the system by repeating the simulations now using gridless electrodes. For the present setup (Fig. 1) we find optimal voltage ratios of $U_{\text {rep }} / U_{\text {extr }}=1.55$ and $U_{\text {rep }} / U_{\text {achr }}=-1.5$ leading to momentum focusing.

\section{Experimental setup}

The technical realization of the HEVMI setup is shown schematically in Fig. 3. Five cylindrical electrodes generate a non-uniform field, which guides the electrons through the spectrometer preserving the information about the $3 \mathrm{D}$ photoelectron momentum vector. An effusive gas injector $(500 \mu \mathrm{m}$ diameter pinhole) is integrated at the center of the repeller electrode. To test the HEVMI spectrometer at low and high energies light sources from a commercial Ti:Sapphire laser system and soft X-rays from the synchrotron radiation source DORIS are applied respectively. Briefly, 


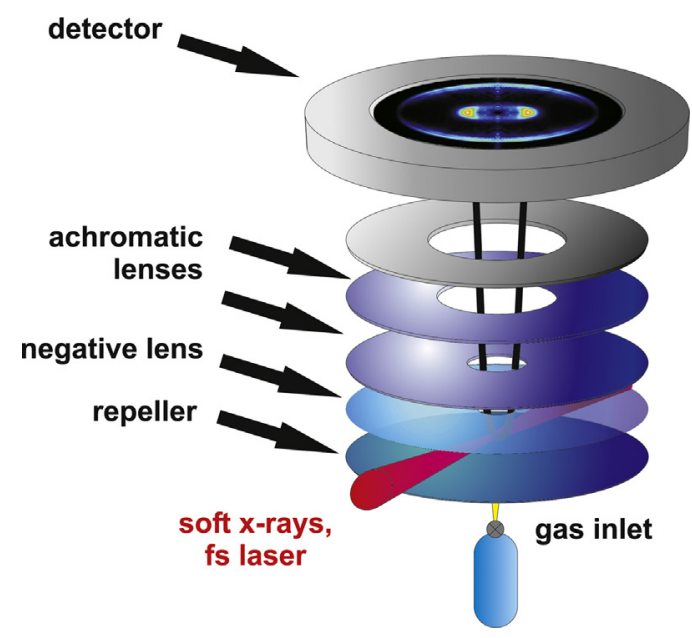

Fig. 3. Schematic view of the High Energy Velocity Map Imaging spectrometer consisting of five cylindrical electrodes. The introduction of a lens of opposite polarity enables to extend the operational energy range far above $1 \mathrm{keV}$. The key point turns out to be the reduction of the expansion time of the Newton spheres (see text for details). The residual gas pressure is about $1.0 \times 10^{-8} \mathrm{mbar}$. During operation the gas load pressure is adjusted to $1.0 \times 10^{-6} \mathrm{mbar}$.

(i) the femtosecond laser system delivers $50 \mathrm{fs}$ pulses at a central wavelength of $800 \mathrm{~nm}\left(\hbar \omega_{L}=1.55 \mathrm{eV}\right)$. By focusing the laser pulse with a $\mathrm{f} / 33$ lens laser intensities of $10^{13} \mathrm{~W} / \mathrm{cm}^{2}$ can be realized allowing for multiphoton ionization of Xe. (ii) For higher electron energies (15-600 eV) experiments have been conducted at the monochromator beam line BW3 at HASYLAB DORIS in Hamburg. We choose $2 \mathrm{~s}$ and $2 \mathrm{p}$ photoemission from atomic neon due to the optimal balance between ionization cross section and electron binding energy [31]. The HEVMI spectrometer was located at a distance of $0.7 \mathrm{~m}$ away from the focus point of the monochromator, which results in an elliptical spot $(162 \mu \mathrm{m}$ in the vertical by $410 \mu \mathrm{m}$ in the horizontal (FWHM)) at the interaction point with a divergence of about 0.1 mrad. Further details can be found elsewhere $[18,25]$. For detection a position-sensitive detector is used consisting of a single multichannel plate (MCP, RoentDek) and a phosphor screen (Proxivision) both of diameter $\phi=80 \mathrm{~mm}$. Sufficient amplification of the photoelectron signal is achieved by applying a voltage of $960 \mathrm{~V}$ at the bottom side of the MCP and $6.5 \mathrm{kV}$ at the front side of the phosphorous screen. To reduce background contributions, as for example signals from detector noise, the MCP voltage was gated using a high-voltage switch (Behlke, HTS-30) with a gate width of $70 \mathrm{~ns}$. The resulting photoelectron images are recorded by a CCD camera (Hamamatsu, ORCA-ER), transferred to a computer and post-processed. Taking into account the cylindrical symmetry of the emitted electron distribution with respect to the polarization axis of the light sources, one can retrieve the complete 3D-information about the photoelectron emission direction from the 2D-picture using, e.g., the onion peeling algorithm [5].

\section{Results and discussion}

\section{Above-Threshold Ionization in xenon}

First performance tests of the HEVMI have been conducted in the low energy range up to $10 \mathrm{eV}$ by means of Above-Threshold Ionization (ATI) from atomic xenon exposed to pulses from the fs laser system. At intensities close to the tunneling regime [15], i.e. $3 \times 10^{13} \mathrm{~W} / \mathrm{cm}^{2}$ for Xe, ATI proceeds via Freeman resonances [10], which largely enhance the ionization probability. The resulting photoemission strongly depends on the on-axis intensity of the laser pulse via the AC Stark shift and leads to specific angular distributions, see [26].
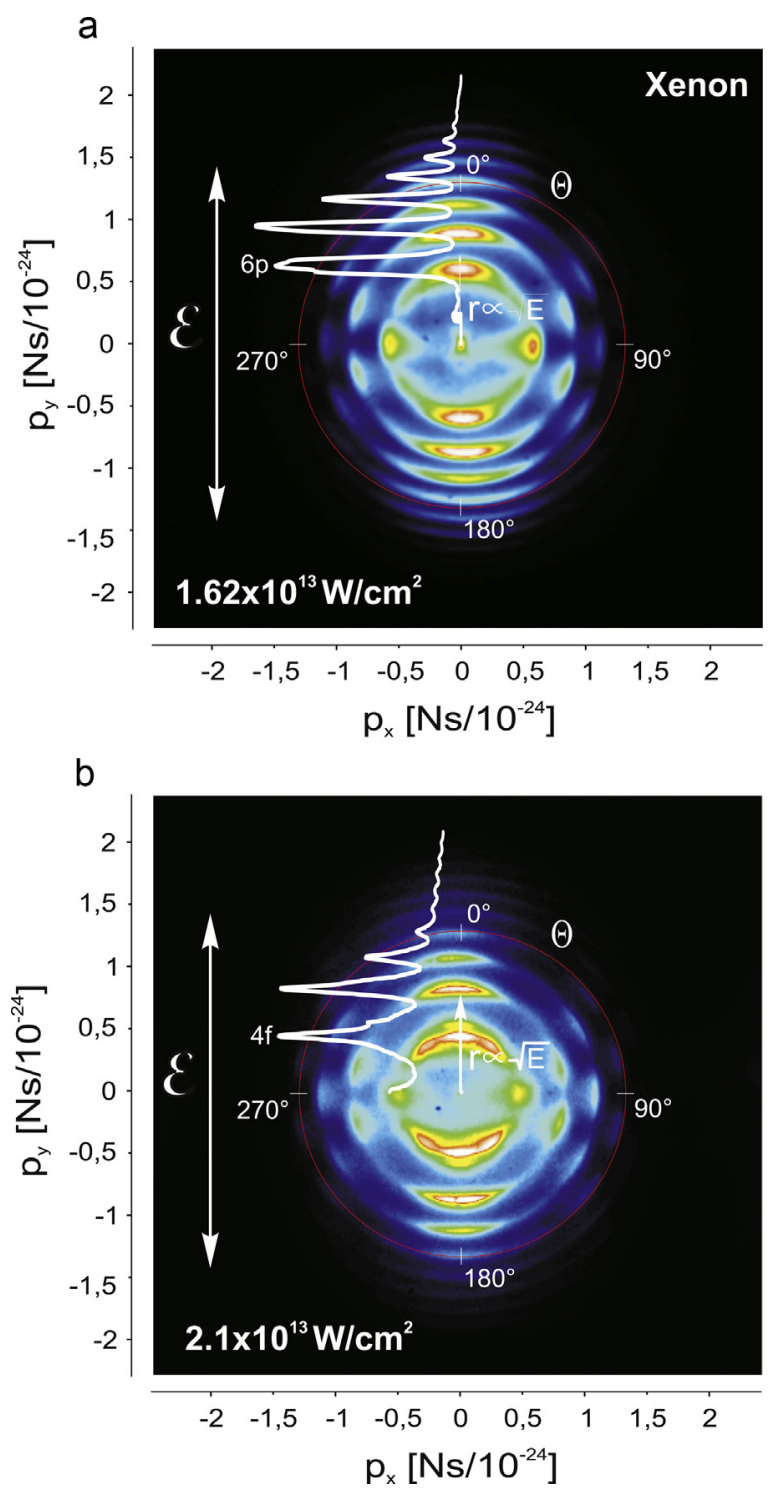

Fig. 4. Raw angular-resolved ATI-spectra of atomic xenon exposed to intense laser fields $\left(\hbar \omega_{L}=1.55 \mathrm{eV}\right.$ ) recorded with the HEVMI spectrometer (laser intensities and polarization axis as depicted in the figure). The ring-like pattern results from the projection of the expanding Newton spheres onto the 2D detector. Note that the spectrometer records momentum instead of energy i.e. $p \sim \sqrt{E_{\text {kin }}}$. The angularintegrated spectra (white lines) show that the ATI features are separated in energy by $\hbar \omega_{L}=1.55 \mathrm{eV}$. As function of laser intensity the peak positions flip from resonant$6 \mathrm{p}$ (top) to resonant-4f (bottom) resulting in a significant change in the angular distribution (see Schyja et al. [26] for further details).

The instantaneous absorption of nine infrared photons is necessary for multiphoton ionization. Additional absorption of photons leads to a characteristic ATI structure in the photoelectron signal. Fig. 4 shows angular distributions observed at two different laser intensities. Note that the ionization potential of $\mathrm{Xe}(12.13 \mathrm{eV})$ is laser intensity dependent and also shifts due to the AC Stark effect. By comparing to measurements conducted by Helm et al. [26], effective laser intensities of $1.62 \times 10^{13} \mathrm{~W} / \mathrm{cm}^{2}$ and $2.1 \times 10^{13} \mathrm{~W} / \mathrm{cm}^{2}$ have been deduced from the figures. Integrating over the angular signals (solid line in the figures) reveals the characteristic laser induced spacings $\left(\hbar \omega_{L}=1.55 \mathrm{eV}\right)$ of the ATI peaks. From the ATI patterns we calibrate the spectrometer in the lower energy range. An interpolation of the peak positions by a second order polynomial enables for an assignment of the electron energy in the entire detection range with a precision of a few percent. 


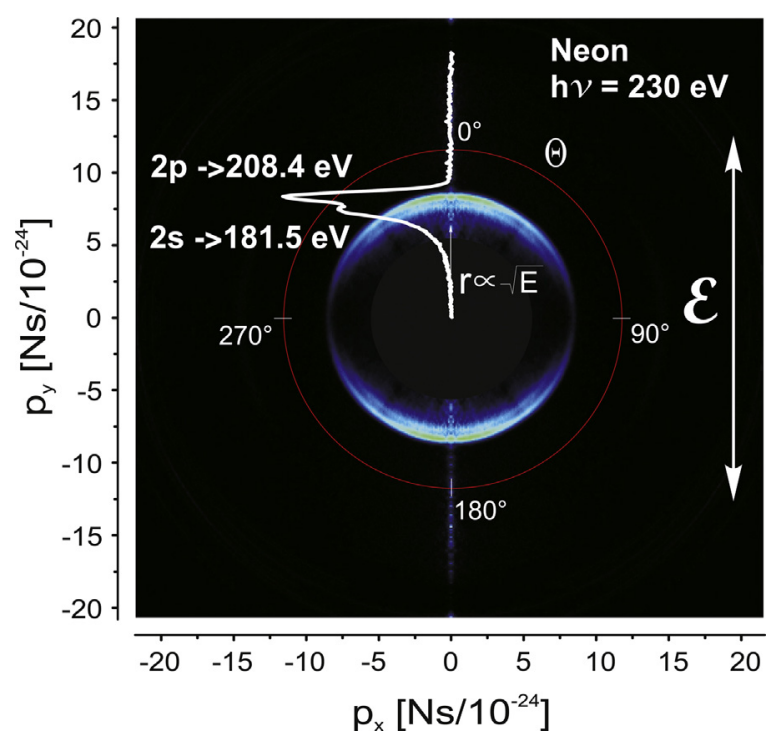

Fig. 5. Deconvoluted HEVMI-spectrum resulting from $230 \mathrm{eV}$ photoionization of $\mathrm{Ne}$ atoms. Two rings separated by an energy of $29.9 \mathrm{eV}$ appear as signals on the position sensitive detector. These are the projections of the 3D electron cloud emitted from $2 \mathrm{p}$ and $2 \mathrm{~s}$ atomic levels. For convenience the center of the detector area is masked.

The ATI features can be used to characterize the mapping properties of the spectrometer [30]. As a measure one can take advantage of the fact that slices of the Newton sphere for a given emission angle with respect to the polarization axis are projected along a straight line in the detector plane [9]. No significant distortion of the angular photoelectron patterns has been obtained. Hence the spectrometer design ensures a sufficient compression of the Newton sphere along the spectrometer symmetry axis. Finally, one can estimate an upper limit of the energy resolution of the HEVMI by analyzing the shape of the Freeman resonances [10] taking into account the actual laser bandwidth of about $70 \mathrm{meV}$. The linewidths (FWHM) of the dominant $4 \mathrm{f}$ and $5 \mathrm{f}$ contributions extracted from the first ring in the ATI pattern of Fig. 4 (bottom) are $130 \mathrm{meV}$ and $80 \mathrm{meV}$, respectively. The extracted numbers are in good agreement with previously reported values of $120 \mathrm{meV}$ and $74 \mathrm{meV}$ obtained by time-of-flight electron spectroscopy [13]. The main constrains on the photoelectron linewidth are imposed by the rather short lifetimes of dressed states leading to the Freeman resonances, typically 1-60 fs [24]. Thus, the short-living states possess natural linewidths on the order of a few tens of meV. The energy resolution of the HEVMI spectrometer is sufficient to resolve the linewidths of the Freeman resonances under study and should therefore at least be comparable to the laser bandwidth, i.e. $\Delta E / E$ to be below $10 \%$.

\section{Soft X-ray photoemission from neon}

The transmission and focus capabilities at higher electron energies have been obtained by means of ionization of gaseous neon with soft X-ray radiation. Single photon absorption leads to the emission from $2 \mathrm{p}$ and 2 s levels having binding energies of 21.7 and $48.5 \mathrm{eV}$, respectively. The electron waves form ring-like patterns on the detector with well-known angular distributions [8], see Fig. 5. Due to the larger $2 \mathrm{p}$ ionization cross section the outermost ring exhibits a higher intensity. At the chosen photon energy of $230 \mathrm{eV}$ the $2 \mathrm{p}$ fine splitting of $\Delta \mathrm{E}=0.1 \mathrm{eV}$ cannot be resolved.

Neon photoemission over a broad energy range has been recorded by scanning the beamline monochromator up to the limit of $600 \mathrm{eV}$. In the studies we adapt the repeller voltage to the photon energy in three steps in order to use the full width of the active

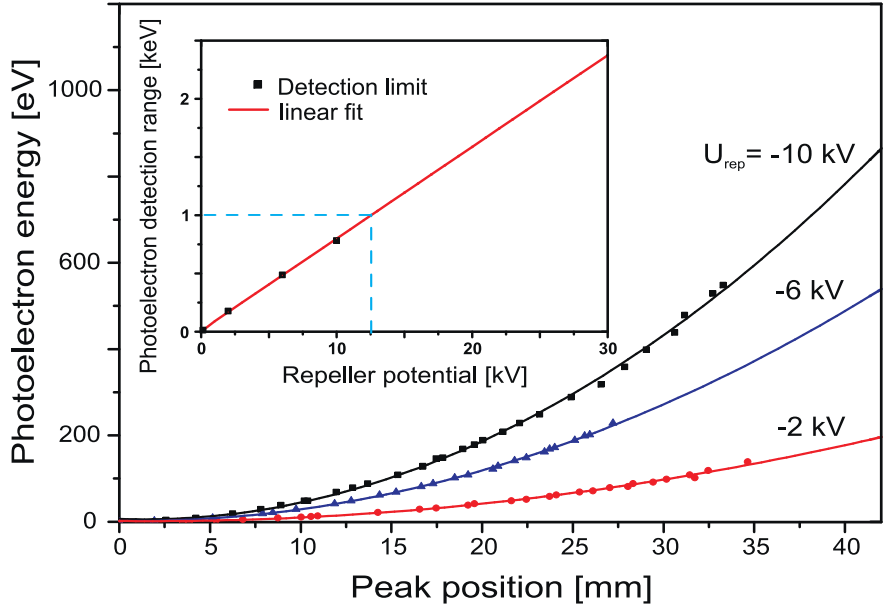

Fig. 6. Calibration curves extracted from soft X-ray studies on neon $2 \mathrm{p}$ photoemission (see Fig. 5) for three different repeller voltage settings (red: $-2 \mathrm{keV}$; blue: $-6 \mathrm{keV}$; black: $-10 \mathrm{keV}$ ). Inset: Extrapolation of a second order polynomial fit for a $80 \mathrm{~mm}$ detector shows that at a repeller voltage of $15 \mathrm{kV}$ full angular resolved energy spectra of electrons with $E_{\text {kin }}>1 \mathrm{keV}$ can be recorded. (For interpretation of the references to color in this figure legend, the reader is referred to the web version of this article.)

detector area. By integrating the angular signals the photoelectron spectra have been extracted (see Fig. 5, white line) and used for calibration. Above $\hbar \omega_{X-\text { ray }}=500 \mathrm{eV}$ the $2 \mathrm{~s}$ and $2 \mathrm{p}$ states cannot be resolved anymore and an averaged energy value has been used in the calculation. This implies that the resolution at higher energies is still about $10 \%$, see Fig. 2 . We emphasize that the experiment has been performed with unfocused radiation. Thus photoemission proceeds along the entire axis of the soft X-ray beam. This leads to a significant photoelectron line broadening due to the volumetric effect, which reduces the effective resolution of the VMI system. The calibration curve as depicted in Fig. 6 can be approximated by a second order polynomial. The procedure assures for an assignment of the electron energy with a precision of about $4 \%$ in further experiments.

The angular distribution mapping capabilities at higher energies is studied by means of the energy-dependent anisotropy parameter $\beta$. For linearly polarized light the angular distribution $I(\Theta)$ can be described by

$I(\Theta)=1+\beta\left(\frac{3}{2} \cos ^{2} \Theta-\frac{1}{2}\right)$

where $\beta$ denotes the anisotropy parameter and $\Theta$ the angle between the direction of the ejected electrons and the polarization axis of the incident light [8]. The results are shown in Fig. 7. Up to $\hbar \omega_{X-r a y}=50 \mathrm{eV}$ the anisotropy parameter increases rapidly starting from -0.2 at low energies and approaching a value of 1.3. The strong dependence of $\beta$ as function of photon energy agrees well with results from a previous study by means of time-of-flight spectroscopy [7]. Moreover, excellent agreement is found with theoretical calculations, which however predict a slightly lower value of $\beta$ for photon energy range above $250 \mathrm{eV}$ [16]. This deviation is caused by the systematically increasing overlap between $2 \mathrm{~s}$ and $2 \mathrm{p}$ contributions due to the focus conditions, which limits the resolution. The experimental studies performed at DORIS synchrotron facility demonstrate the applicability of the new design to record angular-resolved emission patterns in an extended energy regime.

\section{Summary}

An improved version of the Eppink-Parker spectrometer, which includes an additional electrodes of opposite polarity has been 


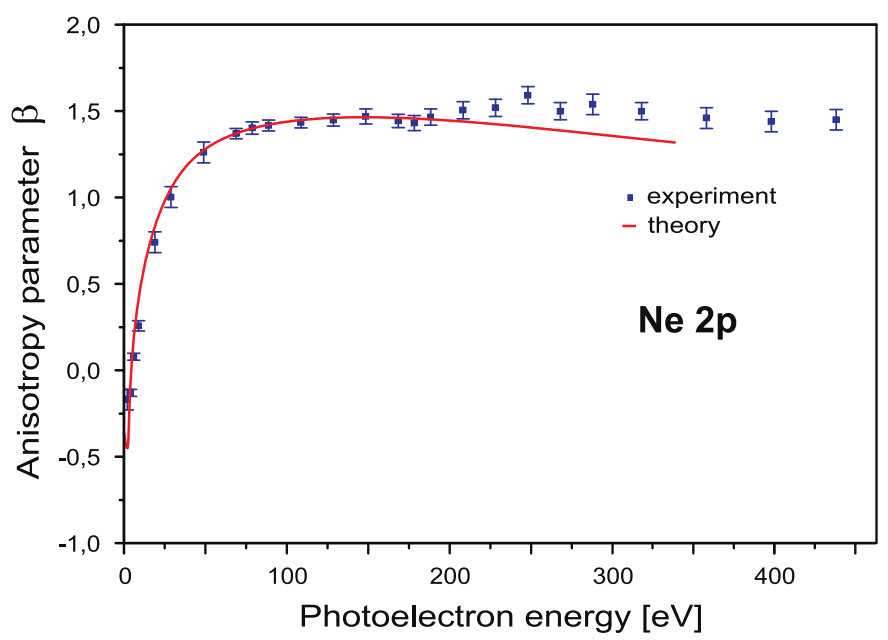

Fig. 7. The anisotropy parameter $\beta$ of $2 \mathrm{p}$-photoemission as a function of XUV photon energy. The strong dependency of the angular distribution (blue squares) is in good agreement with theoretical calculations (solid line) Kennedy and Manson [16]. (For interpretation of the references to color in this figure legend, the reader is referred to the web version of this article.)

constructed and tested up to high kinetic energies. Particle trajectory simulations show that the new compact design enables to map angular distributions in energetic particle emission up to the keV regime applying only moderate repeller voltages, with, at the same time, substantially improved resolution. Experimental studies on Xe ATI and soft X-ray photoemission support the findings from the simulations that the energy and angular resolution remains sufficiently high within the full energy range.

\section{Acknowledgements}

The authors gratefully acknowledge financial support by the Deutsche Forschungsgemeinschaft within the Sonderforschungsbereich 652 . We are obliged to Volkmar Senz, Patrice Oelßner and Jens Bahn for outstanding support during the synchrotron measurements. Thanks to Jens Viefhaus from DORIS III facility at DESY for support during beamtime. One of the authors (S.S.) gratefully acknowledges the Life, Light and Matter Interdisciplinary Faculty of the University of Rostock for scholarship.

\section{References}

[1] B.L.G. Bakker, A.T.J.B. Eppink, D.H. Parker, M.L. Costen, G. Hancock, G.A.D. Ritchie, The sequential two photon dissociation of $\mathrm{NO}$ as a source of aligned N(D-2), N(S-4) and O(P-3) atoms, Chem. Phys. Lett. 283 (1998) 319.

[2] B.L.G. Bakker, D.H. Parker, G. Hancock, G.A.D. Ritchie, Two-photon dissociation of NO near $275 \mathrm{~nm}$ investigated by velocity map imaging, Chem. Phys. Lett. 294 (1998) 565

[3] C. Bartels, C. Hock, J. Huwer, R. Kuhnen, J. Schwöbel, B. von Issendorff, Probing the angular momentum character of the valence orbitals of free sodium nanoclusters, Science 323 (2009) 1323.

[4] C.I. Blaga, J. Xu, A.D. DiChiara, E. Sistrunk, K. Zhang, P. Agostini, T.A. Miller, L.F. DiMauro, C.D. Lin, Imaging ultrafast molecular dynamics with laser-induced electron diffraction, Nature 483 (2012) 194.

[5] C. Bordas, F. Paulig, H. Helm, D.L. Huestis, Photoelectron imaging spectrometry: principle and inversion method, Rev. Sci. Instrum. 67 (1996) 2257.

[6] D.W. Chandler, P.L. Houston, Two-dimensional imaging of state-selected photodissociation products detected by multiphoton ionization, J. Chem. Phys. 87 (1987) 1445.

[7] K. Codling, R.G. Houlgate, W.B. West, P.R. Woodruff, Angular distribution and photoionization measurements on the $2 \mathrm{p}$ and $2 \mathrm{~s}$ electrons in neon, J. Phys. B 9 (1976) L83.
[8] J. Cooper, R.N. Zare, Angular distribution of photoelectrons, J. Chem. Phys. 48 (1968) 942.

[9] A.T.J.B. Eppink, D.H. Parker, Velocity map imaging of ions and electrons using electrostatic lenses: application in photoelectron and photofragment ion imaging of molecular oxygen, Rev. Sci. Instrum. 68 (1997) 3477.

[10] R.R. Freeman, P. Bucksbaum, H. Milchberg, S. Darack, D. Schumacher, M. Geusic Above-threshold ionization with subpicosecond laser-pulses, Phys. Rev. Lett. 59 (1987) 1092

[11] G. Garcia, L. Nahon, C.J. Harding, E.A. Mikajlo, I. Powis, A refocusing modified velocity map imaging electron/ion spectrometer adapted to synchrotron radiation studies, Rev. Sci. Instrum. 76 (2005) 053302.

[12] O. Ghafur, W. Siu, M.F. Kling, M. Drescher, M.J.J. Vrakking, A velocity map imaging detector with an integrated gas injection system, Rev. Sci. Instrum. 80 (2009) 039110.

[13] P. Hansch, M.A. Walker, L.D. Van Woerkom, Eight- and nine-photon resonances in multiphoton ionization of xenon, Phys. Rev. A 57 (1998) R709.

[14] Y. Huismans, A. Rouzee, A. Gijsbertsen, J.H. Jungmann, A.S. Smolkowska P.S.W.M. Logman, F. Lepine, C. Cauchy, S. Zamith, T. Marchenko, J.M. Bakker, G. Berden, B. Redlich, A.F.G. van der Meer, H.G. Muller, W. Vermin, K.J. Schafer, M. Spanner, M.Y. Ivanov, O. Smirnova, D. Bauer, S.V. Popruzhenko, M.J.J. Vrakking, Time-resolved holography with photoelectrons, Nature 331 (2011) 61.

[15] L.V. Keldysh, Ionization in the field of a strong electromagnetic wave, Sov. Phys, JEPT 20 (1965) 1307

[16] D. Kennedy, S. Manson, Photoionization of noble-gases-cross-sections and angular-distributions, Phys. Rev. A 5 (1972) 227.

[17] J.J. Larsen, H. Sakai, C.P. Safvan, I. Wendt-Larsen, H. Stapelfeldt, Aligning molecules with intense nonresonant laser fields, J. Chem. Phys. 111 (1999) 7774.

[18] C.U.S. Larsson, A. Beutler, O. Bjoerneholm, F. Federmann, U. Hahn, A. Riecs T. Verbina, T. Möller, First results from the high-resolution XUV undulator beamline BW3 at Hasylab, Nucl. Instrum. Meth. A 337 (1994) 603.

[19] M. Meyer, P. Radcliffe, T. Tschentscher, J.T. Costello, A.L. Cavalieri, I. Grguras, A.R Maier, R. Kienberger, J. Bozek, C. Bostedt, S. Schorb, R. Coffee, M. Messerschmidt, C. Roedig, E. Sistrunk, L.F. DiMauro, G. Doumy, K. Ueda, S. Wada, S. Düsterer, A.K. Kazansky, N.M. Kabachnik, Angle-resolved electron spectroscopy of laserassisted Auger decay induced by a few-femtosecond X-ray pulse, Phys. Rev. Lett. 108 (2012) 063007.

[20] K. Nagaya, H. Iwayama, H. Murakami, M. Yao, H. Fukuzawa, K. Motomura, K. Ueda, N. Saito, L. Foucar, M. Nagasono, A. Higashiya, M. Yabashi, T. Ishikawa, H. Kimura, H. Ohashi, Investigation of the interaction of xenon cluster with intense EUV-FEL pulses using pulsed cluster beam source and momentum imaging spectrometer, J. Electron Spectrosc. 181 (2010) 125.

[21] H.L. Offerhaus, C. Nicole, F. Lepine, C. Bordas, F. Rosca-Pruna, M.J.J. Vrakking A magnifying lens for velocity map imaging of electrons and ions, Rev. Sci. Instrum. 72 (2001) 3245

[22] J. Passig, R. Irsig, N.X. Truong, T. Fennel, J. Tiggesbäumker, K.H. Meiwes-Broer Nanoplasmonic electron acceleration in silver clusters studied by angularresolved electron spectroscopy, New J. Phys. 14 (2012) 085020.

[23] J.C. Pinare, B. Baguenard, C. Bordas, M. Broyer, Photoelectron imaging spectroscopy of small clusters: evidence for non-Boltzmannian kinetic-energy distribution in thermionic emission, Phys. Rev. Lett. 81 (1998) 2225

[24] R.M. Potvliege, S. Vucic, High-order above-threshold ionization of argon: plateau resonances and the Floquet quasienergy spectrum, Phys. Rev. A 74 (2006) 023412.

[25] R. Reininger, V. Saile, A soft-X-ray grating monochromator for undulator radiation, Nucl. Instrum. Meth. A 288 (1990) 343

[26] V. Schyja, T. Lang, H. Helm, Chanel switching in above-threshold ionization of xenon, Phys. Rev. A 57 (1998) 3692.

[27] Scientific Instrument Services, Industry standard charged particle optics simulation software, 2014 http://www.sisweb.com/simion/simorder.htm

[28] T. Suzuki, L. Wang, H. Kohguchi, Femtosecond time-resolved photoelectron imaging on ultrafast electronic dephasing in an isolated molecules, J. Chem. Phys. 111 (1999) 4859.

[29] A. Vredenborg, W.G. Roeterdink, H.M. Janssen, A photoelectron-photoion coincidence imaging apparatus for femtosecond time-resolved molecular dynamics with electron time-of-flight resolution of $\sigma=18 \mathrm{ps}$ and energy resolution $\Delta \mathrm{E} / \mathrm{E}=3.5 \%$, Rev. Sci. Instrum. 79 (2008) 063108 .

[30] R. Wiehle, B. Witzel, H. Helm, E. Cormier, Dynamics of strong-field abovethreshold ionization of argon: Comparison between experiment and theory, Phys. Rev. A 67 (2003) 063405.

[31] J.J. Yeh, I. Lindau, Atomic subshell photoionization cross-sections and asymmetry parameters, Atom. Data Nucl. Data Tables 32 (1985) 1.

[32] B.L. Yoder, A.H.C. West, B. Schlaeppi, E. Chasovskikh, R. Signorell, A velocity map imaging photoelectron spectrometer for the study of ultrafine aerosols with a table-top VUV laser and Na-doping for particle sizing applied to diethyl ether condensation, J. Chem. Phys. 138 (2013) 138

[33] S. Zherebtsov, T. Fennel, J. Plenge, E. Antonsson, I. Znakovskaya, A. Wirth, O. Herrwerth, F. Süssmann, C. Peltz, I. Ahmad, S.A. Trushin, V. Pervak, S. Karsch, M.J.J. Vrakking, B. Langer, C. Graf, M.I. Stockman, F. Krausz, E. Rühl, M.F. Kling, Controlled near-field enhanced electron acceleration from dielectric nanospheres with intense few-cycle laser fields, Nat. Phys. 7 (2011) 656-662. 